\title{
Some Properties on The $[p, q]-O r d e r$ of Meromorphic Solutions of Homogeneous and Non-homogeneous Linear Differential Equations With Meromorphic Coefficients
}

\author{
Mansouria Saidani, Benharrat Belaïdi*® \\ Department of Mathematics, Laboratory of Pure and Applied Mathematics, University of Mostaganem \\ (UMAB), B. P. 227 Mostaganem, Algeria \\ saidaniman@yahoo.fr, benharrat.belaidi@univ-mosta.dz \\ *Correspondence: benharrat.belaidi@univ-mosta.dz
}

\begin{abstract}
АвSTRACT. In the present paper, we investigate the $[p, q]$-order of solutions of higher order linear differential equations

$$
A_{k}(z) f^{(k)}+A_{k-1}(z) f^{(k-1)}+\cdots+A_{1}(z) f^{\prime}+A_{0}(z) f=0
$$

and

$$
A_{k}(z) f^{(k)}+A_{k-1}(z) f^{(k-1)}+\cdots+A_{1}(z) f^{\prime}+A_{0}(z) f=F(z),
$$

where $A_{0}(z), A_{1}(z), \ldots, A_{k}(z) \not \equiv 0$ and $F(z) \not \equiv 0$ are meromorphic functions of finite $[p, q]$-order.

We improve and extend some results of the authors by using the concept $[p, q]$-order.
\end{abstract}

\section{INTRODUCTION AND MAIN RESULTS}

In this paper, we assume that the reader is familiar with the fundamental results and the standard notations of the Nevanlinna's value distribution theory of meromorphic functions (see [7] , [9] , [14] , [24]). In addition, for any integers $p \geq q \geq 1$ and a meromorphic function $f$ in the whole complex plane, we will use $\rho_{[p, q]}(f), \mu_{[p, q]}(f)$ to denote respectively the $[p, q]$-order and the lower $[p, q]$ order, $\bar{\lambda}_{[p, q]}(f-a)\left(\right.$ or $\left.\lambda_{[p, q]}(f-a)\right)$ to denote the $[p, q]$-convergence exponent of the sequence of distinct a-points (or of a-points) and $\lambda_{[p, q]}\left(\frac{1}{f}\right)$ to denote the $[p, q]$-exponent of convergence of the poles, we refer the reader to see [12] , [15] , [16] and [25]. In particular for $q=1, \rho_{[p, 1]}(f)=\rho_{p}(f)$ is the iterated $p$-order, $\mu_{[p, 1]}(f)=\mu_{p}(f)$ is the iterated lower $p$-order, $\bar{\lambda}_{[p, 1]}(f-a)=\bar{\lambda}_{p}(f, a)$ (or $\left.\lambda_{[p, 1]}(f-a)=\lambda_{p}(f, a)\right)$ is the iterated convergence exponent of the sequence of distinct apoints (or of a-points), $\lambda_{[p, 1]}\left(\frac{1}{f}\right)=\lambda_{p}\left(\frac{1}{f}\right)$ is the iterated exponent of convergence of the poles, see $[7],[11],[13],[14]$ and [24] for notations and definitions.

Received: 6 Sep 2021.

Key words and phrases. linear differential equations; meromorphic functions; $[p, q]$-order; $[p, q]$-exponent of convergence of zeros. 
Several authors have investigated the growth of solutions of second order and higher order homogeneous and non-homogeneous linear differential equations with analytic, entire or meromorphic coefficients, see ([1-3], [6], [8], [11], [13-16], [18] , [20-21], [23], [25]). In the recent years, many authors have studied the complex linear differential equations

$$
\begin{gathered}
f^{(k)}+A_{k-1}(z) f^{(k-1)}+\cdots+A_{1}(z) f^{\prime}+A_{0}(z) f=0, \\
f^{(k)}+A_{k-1}(z) f^{(k-1)}+\cdots+A_{1}(z) f^{\prime}+A_{0}(z) f=F(z),
\end{gathered}
$$

where $A_{0}(z) \not \equiv 0, A_{1}(z), \ldots, A_{k-1}(z)$ and $F(z) \not \equiv 0$ are meromorphic functions of finite iterated p-order. In [2], Belaïdi considered the growth of meromorphic solutions of equations (1.1) and (1.2) with meromorphic coefficients of finite iterated $p$-order and obtained some results which improve and generalize some previous results.

Theorem $\mathrm{A}([2])$ Let $H \subset[0,+\infty)$ be a set with a positive upper density, and let $A_{j}(z)(j=0,1, \ldots$, $k-1)$ be meromorphic functions with finite iterated p-order. If there exist positive constants $\sigma>0, \alpha>0$ such that $\rho=\max \left\{\rho_{p}\left(A_{j}\right): j=1, \ldots, k-1\right\}<\sigma$ and $\left|A_{0}(z)\right| \geq \exp _{p}\left(\alpha r^{\sigma}\right)$ as $|z|=r \in H, r \rightarrow+\infty$, then every meromorphic solution $f \not \equiv 0$ of equation (1.1) satisfies

$$
\mu_{p}(f)=\rho_{p}(f)=+\infty, \rho_{p+1}(f) \geq \sigma .
$$

Furthermore, if $\lambda_{p}\left(\frac{1}{f}\right)<\infty$, then $i(f)=p+1$ and

$$
\sigma \leq \rho_{p+1}(f) \leq \rho_{p}\left(A_{0}\right) .
$$

Theorem B ([2]) Let $H \subset[0,+\infty)$ be a set with a positive upper density, and let $A_{j}(z)(j=$ $0,1, \ldots, k-1)$ and $F(z) \not \equiv 0$ be meromorphic functions with finite iterated p-order. If there exist positive constants $\sigma>0, \alpha>0$ such that $\left|A_{0}(z)\right| \geq \exp _{p}\left(\alpha r^{\sigma}\right)$ as $|z|=r \in H, r \rightarrow+\infty$, and $\rho=\max \left\{\rho_{p}\left(A_{j}\right)(j=1, \ldots, k-1), \rho_{p}(F)\right\}<\sigma$, then every meromorphic solution of equation (1.2) with $\lambda_{p}\left(\frac{1}{f}\right)<\sigma$ satisfies

$$
\bar{\lambda}_{p}(f)=\lambda_{p}(f)=\rho_{p}(f)=\infty, \bar{\lambda}_{p+1}(f)=\lambda_{p+1}(f)=\rho_{p+1}(f) .
$$

Furthermore, if $\lambda_{p}\left(\frac{1}{f}\right)<\min \left\{\mu_{p}(f), \sigma\right\}$, then $i(f)=p+1$ and

$$
\bar{\lambda}_{p+1}(f)=\lambda_{p+1}(f)=\rho_{p+1}(f) \leq \rho_{p}\left(A_{0}\right) .
$$

Recently, in [18] the authors have studied the growth of solutions of the equations (1.1) and (1.2) when $A_{s}(z)$ to dominate all other coefficients and they got some results about $\rho_{p+1}(f)$ as follows.

Theorem $C([18])$ Let $H \subset(1,+\infty)$ be a set with a positive upper logarithmic density (or $m_{l}(H)=$ $+\infty)$, and let $A_{j}(z)(j=0,1, \ldots, k-1)$ be meromorphic functions with finite iterated $p$-order. 
If there exist positive constants $\sigma>0, \alpha>0$ and an integer $s, 0 \leq s \leq k-1$, such that $\left|A_{s}(z)\right| \geq \exp _{p}\left(\alpha r^{\sigma}\right)$ as $|z|=r \in H, r \rightarrow+\infty$, and $\rho=\max \left\{\rho_{p}\left(A_{j}\right)(j \neq s)\right\}<\sigma$, then every non-transcendental meromorphic solution $f \not \equiv 0$ of (1.1) is a polynomial with $\operatorname{deg} f \leq s-1$ and every transcendental meromorphic solution $f$ of $(1.1)$ with $\lambda_{p}\left(\frac{1}{f}\right)<\mu_{p}(f)$ satisfies $i(f)=p+1$

$$
\mu_{p}(f)=\rho_{p}(f)=+\infty
$$

and

$$
\sigma \leq \rho_{p+1}(f) \leq \rho_{p}\left(A_{s}\right)
$$

Theorem $\mathbf{D}([18])$ Let $H \subset(1,+\infty)$ be a set with a positive upper logarithmic density (or $m_{l}(H)=$ $+\infty)$, and let $A_{j}(z)(j=0,1, \ldots, k-1)$ and $F(z) \not \equiv 0$ be meromorphic functions with finite iterated p-order. If there exist positive constants $\sigma>0, \alpha>0$ and an integer $s, 0 \leq s \leq k-1$, such that $\left|A_{s}(z)\right| \geq \exp _{p}\left(\alpha r^{\sigma}\right)$ as $|z|=r \in H, r \rightarrow+\infty$, and $\max \left\{\rho_{p}\left(A_{j}\right)(j \neq s), \rho_{p}(F)\right\}<\sigma$, then every non-transcendental meromorphic solution $f$ of $(1.2)$ is a polynomial with $\operatorname{deg} f \leq s-1$ and every transcendental meromorphic solution $f$ of (1.2) with $\lambda_{p}\left(\frac{1}{f}\right)<\min \left\{\sigma, \mu_{p}(f)\right\}$ satisfies $i(f)=p+1$

$$
\bar{\lambda}_{p}(f)=\lambda_{p}(f)=\rho_{p}(f)=\mu_{p}(f)=+\infty
$$

and

$$
\sigma \leq \bar{\lambda}_{p+1}(f)=\lambda_{p+1}(f)=\rho_{p+1}(f) \leq \rho_{p}\left(A_{s}\right)
$$

Thus, the following question arises: can we have the same properties as in Theorems $C$ and $\mathrm{D}$ for the solutions of equations

$$
A_{k}(z) f^{(k)}+A_{k-1}(z) f^{(k-1)}+\cdots+A_{1}(z) f^{\prime}+A_{0}(z) f=0
$$

and

$$
A_{k}(z) f^{(k)}+A_{k-1}(z) f^{(k-1)}+\cdots+A_{1}(z) f^{\prime}+A_{0}(z) f=F(z),
$$

when the coefficients $A_{j}(j=0,1, \ldots, k)$ are of $[p, q]$-order? In this paper, we proceed this way and we obtain the following results.

Theorem 1.1 Let $H \subset(1,+\infty)$ be a set with a positive upper logarithmic density (or $m_{l}(H)=$ $+\infty)$ and let $A_{j}(z)(j=0,1, \ldots, k)$ with $A_{k}(z) \not \equiv 0$ be meromorphic functions with finite $[p, q]-$ order. If there exist a positive constant $\sigma>0$ and an integer $s, 0 \leq s \leq k$, such that for sufficiently small $\varepsilon>0$, we have $\left|A_{s}(z)\right| \geq \exp _{p+1}\left\{(\sigma-\varepsilon) \log _{q} r\right\}$ as $|z|=r \in H, r \rightarrow+\infty$ and $\rho=\max \left\{\rho_{[p, q]}\left(A_{j}\right)(j \neq s)\right\}<\sigma$, then every non-transcendental meromorphic solution $f \not \equiv 0$ of (1.3) is a polynomial with $\operatorname{deg} f \leq s-1$ and every transcendental meromorphic solution $f$ of (1.3) with $\lambda_{[p, q]}\left(\frac{1}{f}\right)<\mu_{[p, q]}(f)$ satisfies

$$
\rho_{[p, q]}(f)=\mu_{[p, q]}(f)=+\infty, \sigma \leq \rho_{[p+1, q]}(f) \leq \rho_{[p, q]}\left(A_{s}\right) .
$$

Remark 1.1 Putting $A_{k}(z) \equiv 1$ and $q=1$ in Theorem 1.1, we obtain Theorem C. 
Corollary 1.1 Under the hypotheses of Theorem 1.1, suppose further that $\varphi$ is a transcendental meromorphic function satisfying $\rho_{[p+1, q]}(\varphi)<\sigma$. Then, every transcendental meromorphic solution $f$ of equation (1.3) with $\lambda_{[p, q]}\left(\frac{1}{f}\right)<\mu_{[p, q]}(f)$ satisfies

$$
\begin{gathered}
\sigma \leq \bar{\lambda}_{[p+1, q]}(f-\varphi)=\lambda_{[p+1, q]}(f-\varphi) \\
=\rho_{[p+1, q]}(f-\varphi)=\rho_{[p+1, q]}(f) \leq \rho_{[p, q]}\left(A_{s}\right) .
\end{gathered}
$$

Considering the non-homogeneous linear differential equation (1.4), we obtain the following results.

Theorem 1.2 Let $H \subset(1,+\infty)$ be a set with a positive upper logarithmic density (or $m_{l}(H)=$ $+\infty)$, and let $A_{j}(z)(j=0,1, \ldots, k)$ with $A_{k}(z) \not \equiv 0$ and $F(z) \not \equiv 0$ be meromorphic functions with finite $[p, q]$-order. If there exist a positive constant $\sigma>0$ and an integer $s, 0 \leq s \leq k$, such that for sufficiently small $\varepsilon>0$, we have $\left|A_{s}(z)\right| \geq \exp _{p+1}\left\{(\sigma-\varepsilon) \log _{q} r\right\}$ as $|z|=r \in H, r \rightarrow+\infty$ and $\max \left\{\rho_{[p, q]}\left(A_{j}\right)(j \neq s), \rho_{[p, q]}(F)\right\}<\sigma$, then every non-transcendental meromorphic solution $f$ of (1.4) is a polynomial with $\operatorname{deg} f \leq s-1$ and every transcendental meromorphic solution $f$ of (1.4) with $\lambda_{[p, q]}\left(\frac{1}{f}\right)<\min \left\{\sigma, \mu_{[p, q]}(f)\right\}$ satisfies

$$
\bar{\lambda}_{[p, q]}(f)=\lambda_{[p, q]}(f)=\rho_{[p, q]}(f)=\mu_{[p, q]}(f)=+\infty
$$

and

$$
\sigma \leq \bar{\lambda}_{[p+1, q]}(f)=\lambda_{[p+1, q]}(f)=\rho_{[p+1, q]}(f) \leq \rho_{[p, q]}\left(A_{s}\right)
$$

Remark 1.2 Putting $A_{k}(z) \equiv 1$ and $q=1$ in Theorem 1.2, we obtain Theorem D.

Corollary 1.2 Let $A_{j}(z)(j=0,1, \ldots, k), F(z), H$ satisfy all the hypotheses of Theorem 1.2, and let $\varphi$ be a transcendental meromorphic function satisfying $\rho_{[p+1, q]}(\varphi)<\sigma$. Then, every transcendental meromorphic solution $f$ with $\lambda_{[p, q]}\left(\frac{1}{f}\right)<\min \left\{\sigma, \mu_{[p, q]}(f)\right\}$ of equation (1.4) satisfies $\sigma \leq \bar{\lambda}_{[p+1, q]}(f-\varphi)=\lambda_{[p+1, q]}(f-\varphi)=\rho_{[p+1, q]}(f-\varphi) \leq \rho_{[p, q]}\left(A_{s}\right)$.

Remark 1.3 In $[17,19]$, the authors have studied the growth and the oscillation of solutions of equations (1.3) and (1.4) when the coefficients $A_{j}(z)(j=0,1, \ldots, k)$ and $F(z)$ are entire functions of iterated $p$-order or of $[p, q]$-order. However, in the present paper the coefficients $A_{j}(z)$ $(j=0,1, \ldots, k)$ and $F(z)$ are meromorphic functions with reduction of the hypotheses in Theorems 1.1 and 1.2. So, this article may be understood as an extension and an improvement of $[17,19]$. 


\section{SOME AUXILIARY LEMMAS}

In order to prove our theorems, we need the following definition, proposition and lemmas. The Lebesgue linear measure of a set $E \subset[0,+\infty)$ is $m(E)=\int_{E} d t$, and the logarithmic measure of a set $F \subset[1,+\infty)$ is $m_{l}(F)=\int_{F} \frac{d t}{t}$. The upper density of $E \subset[0,+\infty)$ is given by

$$
\overline{\operatorname{dens}}(E)=\limsup _{r \rightarrow \infty} \frac{m(E \cap[0, r])}{r}
$$

and the upper logarithmic density of the set $F \subset[1,+\infty)$ is defined by

$$
\overline{\log d e n s}(F)=\limsup _{r \longrightarrow+\infty} \frac{m_{l}(F \cap[1, r])}{\log r} .
$$

Proposition 2.1 ([2]) For all $H \subset(1,+\infty)$ the following statements hold:

(i) If $m_{l}(H)=+\infty$, then $m(H)=+\infty$;

(ii) If $\overline{\text { dens }}(H)>0$, then $m(H)=+\infty$;

(iii) If $\overline{\log d e n s}(H)>0$, then $m_{l}(H)=+\infty$.

Lemma 2.1 ([5]) Let $f$ be a transcendental meromorphic function in the plane, and let $\alpha>1$ be a given constant. Then, there exist a set $E_{1} \subset(1,+\infty)$ that has a finite logarithmic measure, and a constant $B>0$ depending only on $\alpha$ and $(i, j)((i, j)$ positive integers with $i>j)$ such that for all $z$ with $|z|=r \notin[0,1] \cup E_{1}$, we have

$$
\left|\frac{f^{(i)}(z)}{f^{(j)}(z)}\right| \leq B\left(\frac{T(\alpha r, f)}{r}\left(\log ^{\alpha} r\right) \log T(\alpha r, f)\right)^{i-j} .
$$

Lemma 2.2 ([4]) Let $p \geq q \geq 1$ be integers and $g$ be an entire function such that $\rho_{[p, q]}(g)<+\infty$. Then, there exist entire functions $u(z)$ and $v(z)$ such that

$$
\begin{gathered}
g(z)=u(z) e^{v(z)} \\
\rho_{[p, q]}(g)=\max \left\{\rho_{[p, q]}(u), \rho_{[p, q]}\left(e^{v(z)}\right)\right\}
\end{gathered}
$$

and

$$
\rho_{[p, q]}(u)=\limsup _{r \rightarrow+\infty} \frac{\log _{p} N\left(r, \frac{1}{g}\right)}{\log _{q} r} .
$$

Moreover, for any given $\varepsilon>0$, we have

$$
|u(z)| \geq \exp \left\{-\exp _{p}\left\{\left(\rho_{[p, q]}(u)+\varepsilon\right) \log _{q} r\right\}\right\} \quad\left(r \notin E_{2}\right),
$$

where $E_{2} \subset(1,+\infty)$ is a set of $r$ of finite linear measure. 
Lemma 2.3 Let $p \geq q \geq 1$ be integers. Suppose that $f$ is a meromorphic function such that $\rho_{[p, q]}(f)<+\infty$. Then, there exist entire functions $u_{1}(z), u_{2}(z)$ and $v(z)$ such that

$$
f(z)=\frac{u_{1}(z) e^{v(z)}}{u_{2}(z)}
$$

and

$$
\rho_{[p, q]}(f)=\max \left\{\rho_{[p, q]}\left(u_{1}\right), \rho_{[p, q]}\left(u_{2}\right), \rho_{[p, q]}\left(e^{v(z)}\right)\right\} .
$$

Moreover, for any given $\varepsilon>0$, we have

$$
\begin{aligned}
& \exp \left\{-\exp _{p}\left\{\left(\rho_{(p, q)}(f)+\varepsilon\right) \log _{q} r\right\}\right\} \leq|f(z)| \\
& \leq \exp _{p+1}\left\{\left(\rho_{(p, q)}(f)+\varepsilon\right) \log _{q} r\right\} \quad\left(r \notin E_{3}\right),
\end{aligned}
$$

where $E_{3} \subset(1,+\infty)$ is a set of $r$ of finite linear measure.

Proof. When $p \geq q=1$, the lemma is due to Tu and Long [21]. Thus, we assume that $p>q>1$ or $p=q>1$. By Hadamard factorization theorem, we can write $f$ as $f(z)=\frac{g(z)}{d(z)}$, where $g(z)$ and $d(z)$ are entire functions satisfying

$$
\mu_{[p, q]}(g)=\mu_{[p, q]}(f)=\mu \leq \rho_{[p, q]}(f)=\rho_{[p, q]}(g)<+\infty
$$

and

$$
\lambda_{[p, q]}(d)=\rho_{[p, q]}(d)=\lambda_{[p, q]}\left(\frac{1}{f}\right)<\mu .
$$

By Lemma 2.2, there exist entire functions $u(z)$ and $v(z)$ such that

$$
g(z)=u(z) e^{v(z)}, \rho_{[p, q]}(g)=\max \left\{\rho_{[p, q]}(u), \rho_{[p, q]}\left(e^{v(z)}\right)\right\} .
$$

So, there exist entire functions $u(z), v(z)$ and $d(z)$ such that

$$
f(z)=\frac{u(z) e^{v(z)}}{d(z)}
$$

and

$$
\rho_{[p, q]}(f)=\max \left\{\rho_{[p, q]}(u), \rho_{[p, q]}(d), \rho_{[p, q]}\left(e^{v(z)}\right)\right\} .
$$

Thus (2.1) and (2.2) hold. Set $f(z)=\frac{u_{1}(z) e^{v(z)}}{u_{2}(z)}$, where $u_{1}(z), u_{2}(z)$ are the canonical products formed with the zeros and poles of $f$ respectively. By the definition of $[p, q]$-order, for sufficiently large $r$ and any given $\varepsilon>0$, we have

$$
\begin{aligned}
& \left|u_{1}(z)\right| \leq \exp _{p+1}\left\{\left(\rho_{[p, q]}\left(u_{1}\right)+\frac{\varepsilon}{3}\right) \log _{q} r\right\}, \\
& \left|u_{2}(z)\right| \leq \exp _{p+1}\left\{\left(\rho_{[p, q]}\left(u_{2}\right)+\frac{\varepsilon}{3}\right) \log _{q} r\right\} .
\end{aligned}
$$

Since $\max \left\{\rho_{[p, q]}\left(u_{1}\right), \rho_{[p, q]}\left(u_{2}\right), \rho_{[p, q]}\left(e^{v(z)}\right)\right\}=\rho_{[p, q]}(f)$, then we obtain

$$
\begin{aligned}
& \left|u_{1}(z)\right| \leq \exp _{p+1}\left\{\left(\rho_{[p, q]}(f)+\frac{\varepsilon}{3}\right) \log _{q} r\right\}, \\
& \left|u_{2}(z)\right| \leq \exp _{p+1}\left\{\left(\rho_{[p, q]}(f)+\frac{\varepsilon}{3}\right) \log _{q} r\right\} .
\end{aligned}
$$




$$
\left|e^{v(z)}\right| \leq \exp _{p+1}\left\{\left(\rho_{[p, q]}(f)+\frac{\varepsilon}{3}\right) \log _{q} r\right\} .
$$

By Lemma 2.2, there exists a set $E_{3} \subset(1,+\infty)$ of $r$ with a finite linear measure such that for any given $\varepsilon>0$, we have

$$
\begin{aligned}
& \left|u_{1}(z)\right| \geq \exp \left\{-\exp _{p}\left\{\left(\rho_{[p, q]}\left(u_{1}\right)+\frac{\varepsilon}{3}\right) \log _{q} r\right\}\right\} \\
\geq & \exp \left\{-\exp _{p}\left\{\left(\rho_{[p, q]}(f)+\frac{\varepsilon}{3}\right) \log _{q} r\right\}\right\}, \quad\left(r \notin E_{3}\right), \\
& \left|u_{2}(z)\right| \geq \exp \left\{-\exp _{p}\left\{\left(\rho_{[p, q]}\left(u_{2}\right)+\frac{\varepsilon}{3}\right) \log _{q} r\right\}\right\} \\
\geq & \exp \left\{-\exp _{p}\left\{\left(\rho_{[p, q]}(f)+\frac{\varepsilon}{3}\right) \log _{q} r\right\}\right\}, \quad\left(r \notin E_{3}\right) .
\end{aligned}
$$

Then, by using (2.5) , (2.7) and (2.9), we obtain for sufficiently large $r \notin E_{3}$ and any given $\varepsilon>0$

$$
\begin{gathered}
|f(z)|=\frac{\left|u_{1}(z)\right|\left|e^{v(z)}\right|}{\left|u_{2}(z)\right|} \\
\leq \frac{\exp _{p+1}\left\{\left(\rho_{[p, q]}(f)+\frac{\varepsilon}{3}\right) \log _{q} r\right\} \exp _{p+1}\left\{\left(\rho_{[p, q]}(f)+\frac{\varepsilon}{3}\right) \log _{q} r\right\}}{\exp \left\{-\exp _{p}\left\{\left(\rho_{[p, q]}(f)+\frac{\varepsilon}{3}\right) \log _{q} r\right\}\right\}} \\
\leq \exp _{p+1}\left\{\left(\rho_{[p, q]}(f)+\varepsilon\right) \log _{q} r\right\} .
\end{gathered}
$$

On the other hand, we have $\rho_{[p-1, q]}(v)=\rho_{[p, q]}\left(e^{v(z)}\right) \leq \rho_{[p, q]}(f)$ and $\left|e^{v(z)}\right| \geq e^{-|v(z)|}$. Making use of the definition of $[p, q]$-order, we obtain

$$
\begin{aligned}
&|v(z)| \leq M(r, v) \leq \exp _{p}\left\{\left(\rho_{(p-1, q)}(v)+\frac{\varepsilon}{3}\right) \log _{q} r\right\} \\
& \leq \exp _{p}\left\{\left(\rho_{[p, q]}(f)+\frac{\varepsilon}{3}\right) \log _{q} r\right\} .
\end{aligned}
$$

Then, for sufficiently large $r$ and any given $\varepsilon>0$, we have

$$
\left|e^{v(z)}\right| \geq e^{-|v(z)|} \geq \exp \left\{-\exp _{p}\left\{\left(\rho_{[p, q]}(f)+\frac{\varepsilon}{3}\right) \log _{q} r\right\}\right\}
$$

By (2.6), (2.8) and (2.11), we can easily obtain

$$
\begin{gathered}
|f(z)|=\frac{\left|u_{1}(z)\right|\left|e^{v(z)}\right|}{\left|u_{2}(z)\right|} \\
\geq \frac{\exp \left\{-\exp _{p}\left\{\left(\rho_{[p, q]}(f)+\frac{\varepsilon}{3}\right) \log _{q} r\right\}\right\} \exp \left\{-\exp _{p}\left\{\left(\rho_{[p, q]}(f)+\frac{\varepsilon}{3}\right) \log _{q} r\right\}\right\}}{\exp _{p+1}\left\{\left(\rho_{[p, q]}(f)+\frac{\varepsilon}{3}\right) \log _{q} r\right\}} . \\
=\exp \left\{-3 \exp _{p}\left\{\left(\rho_{[p, q]}(f)+\frac{\varepsilon}{3}\right) \log _{q} r\right\}\right\} \\
\geq \exp \left\{-\exp _{p}\left\{\left(\rho_{[p, q]}(f)+\varepsilon\right) \log _{q} r\right\}\right\} .
\end{gathered}
$$

Thus, we complete the proof of Lemma 2.3.

Lemma 2.4 Under the assumptions of Theorem 1.1 or Theorem 1.2, we have $\rho_{[p, q]}\left(A_{s}\right)=\beta \geq \sigma$. 
Proof. Assume that $\rho_{[p, q]}\left(A_{s}\right)=\beta<\sigma$. According to the hypotheses of Theorems 1.1 or 1.2, there exists a positive constant $\sigma>0$ such that for sufficiently small $\varepsilon>0$, we have

$$
\left|A_{s}(z)\right| \geq \exp _{p+1}\left\{(\sigma-\varepsilon) \log _{q} r\right\}
$$

as $|z|=r \in H, r \rightarrow+\infty$, where $H \subset(1,+\infty)$ is a set with a positive upper logarithmic density (by Proposition 2.1, we have $\left.m_{l}(H)=+\infty\right)$. By Lemma 2.3, we can find a set $E_{3} \subset(1,+\infty)$ that has finite linear measure (and so of finite logarithmic measure) such that when $|z|=r \notin E_{3}$, we have for any given $\varepsilon(0<2 \varepsilon<\sigma-\beta)$

$$
\left|A_{s}(z)\right| \leq \exp _{p+1}\left\{(\beta+\varepsilon) \log _{q} r\right\} .
$$

By (2.12) and (2.13), we obtain for $|z|=r \in H \backslash E_{3}, r \rightarrow+\infty$

$$
\exp _{p+1}\left\{(\sigma-\varepsilon) \log _{q} r\right\} \leq\left|A_{s}(z)\right| \leq \exp _{p+1}\left\{(\beta+\varepsilon) \log _{q} r\right\}
$$

and by $\varepsilon(0<2 \varepsilon<\sigma-\beta)$ this is a contradiction. Hence $\rho_{[p, q]}\left(A_{s}\right)=\beta \geq \sigma$.

Lemma 2.5 (Wiman-Valiron, [10], [22]) Let $f$ be a transcendental entire function, and let $z$ be a point with $|z|=r$ at which $|f(z)|=M(r, f)$. Then the estimation

$$
\frac{f^{(j)}(z)}{f(z)}=\left(\frac{\nu_{f}(r)}{z}\right)^{j}(1+o(1))(j \geq 1 \text { is an integer })
$$

holds for all $|z|$ outside a set $E_{4}$ of $r$ of finite logarithmic measure, where $\nu_{f}(r)$ is the central index of $f$.

Lemma 2.6 ([12]) Let $f$ be an entire function of $[p, q]$-order and let $\nu_{f}(r)$ be the central index of $f$. Then

$$
\rho_{[p, q]}(f)=\limsup _{r \rightarrow+\infty} \frac{\log _{p} \nu_{f}(r)}{\log _{q} r}, \mu_{[p, q]}(f)=\liminf _{r \rightarrow+\infty} \frac{\log _{p} \nu_{f}(r)}{\log _{q} r} .
$$

The following two lemmas were given in [4] without proof, so for the convenience of the reader, we prove them.

Lemma 2.7 Let $f(z)=\frac{g(z)}{d(z)}$ be a meromorphic function, where $g(z), d(z)$ are entire functions satisfying $\mu_{[p, q]}(g)=\mu_{[p, q]}(f)=\mu \leq \rho_{[p, q]}(f)=\rho_{[p, q]}(g) \leq+\infty$ and $\lambda_{[p, q]}(d)=\rho_{[p, q]}(d)=$ $\beta=\lambda_{[p, q]}\left(\frac{1}{f}\right)<\mu$. Then, there exists a set $E_{5} \subset(1,+\infty)$ of finite logarithmic measure such that for all $|z|=r \notin[0,1] \cup E_{5}$ and $|g(z)|=M(r, g)$, we have

$$
\frac{f^{(n)}(z)}{f(z)}=\left(\frac{\nu_{g}(r)}{z}\right)^{n}(1+o(1)), n \in \mathbb{N}
$$

where $\nu_{g}(r)$ denote the central index of $g$. 
Proof. By mathematical induction, we obtain

$$
f^{(n)}=\frac{g^{(n)}}{d}+\sum_{j=0}^{n-1} \frac{g^{(j)}}{d} \sum_{\left(j_{1} \ldots j_{n}\right)} C_{j j_{1} \ldots j_{n}}\left(\frac{d^{\prime}}{d}\right)^{j_{1}} \times \cdots \times\left(\frac{d^{(n)}}{d}\right)^{j_{n}},
$$

where $C_{j j_{1} \ldots j_{n}}$ are constants and $j+j_{1}+2 j_{2}+\cdots+n j_{n}=n$. Hence

$$
\frac{f^{(n)}}{f}=\frac{g^{(n)}}{g}+\sum_{j=0}^{n-1} \frac{g^{(j)}}{g} \sum_{\left(j_{1} \ldots j_{n}\right)} C_{j j_{1} \ldots j_{n}}\left(\frac{d^{\prime}}{d}\right)^{j_{1}} \times \cdots \times\left(\frac{d^{(n)}}{d}\right)^{j_{n}} .
$$

From Lemma 2.5, there exists a set $E_{4} \subset(1,+\infty)$ with finite logarithmic measure such that for a point $z$ satisfying $|z|=r \notin E_{4}$ and $|g(z)|=M(r, g)$, we have

$$
\frac{g^{(j)}(z)}{g(z)}=\left(\frac{\nu_{g}(r)}{z}\right)^{j}(1+o(1))(j=1,2, \ldots, n),
$$

where $\nu_{g}(r)$ is the central index of $g$. Substituting (2.16) into (2.15) yields

$$
\begin{gathered}
\frac{f^{(n)}(z)}{f(z)}=\left(\frac{\nu_{g}(r)}{z}\right)^{n}[(1+o(1)) \\
\left.+\sum_{j=0}^{n-1}\left(\frac{\nu_{g}(r)}{z}\right)^{j-n}(1+o(1)) \sum_{\left(j_{1} \ldots j_{n}\right)} C_{j j_{1} \ldots j_{n}}\left(\frac{d^{\prime}}{d}\right)^{j_{1}} \times \cdots \times\left(\frac{d^{(n)}}{d}\right)^{j_{n}}\right] .
\end{gathered}
$$

Since $\rho_{[p, q]}(d)=\beta<\mu$, then for any given $\varepsilon(0<2 \varepsilon<\mu-\beta)$ and sufficiently large $r$, we have

$$
T(r, d) \leq \exp _{p}\left\{\left(\beta+\frac{\varepsilon}{2}\right) \log _{q} r\right\}
$$

By using Lemma 2.1, for $\alpha=2$, there exist a set $E_{1} \subset(1,+\infty)$ with $m_{l}\left(E_{1}\right)<\infty$ and a constant $B>0$, such that for all $z$ satisfying $|z|=r \notin[0,1] \cup E_{1}$, we have

$$
\begin{aligned}
\left|\frac{d^{(m)}(z)}{d(z)}\right| \leq & B[T(2 r, d)]^{m+1} \leq B\left[\exp _{p}\left\{\left(\beta+\frac{\varepsilon}{2}\right) \log _{q}(2 r)\right\}\right]^{m+1} \\
& \leq \exp _{p}\left\{(\beta+\varepsilon) \log _{q} r\right\}^{m}, m=1,2, \ldots, n .
\end{aligned}
$$

By Lemma 2.6 and $\mu_{[p, q]}(g)=\mu_{[p, q]}(f)=\mu$, it follows that

$$
\nu_{g}(r)>\exp _{p}\left\{(\mu-\varepsilon) \log _{q} r\right\}
$$

for sufficiently large $r$. Thus, by using $j_{1}+2 j_{2}+\cdots+n j_{n}=n-j$, we obtain

$$
\begin{aligned}
\mid\left(\frac{\nu_{g}(r)}{z}\right)^{j-n}\left(\frac{d^{\prime}}{d}\right)^{j_{1}} & \times \cdots \times\left(\frac{d^{(n)}}{d}\right)^{j_{n}} \mid \leq\left[\frac{\exp _{p}\left\{(\mu-\varepsilon) \log _{q} r\right\}}{r}\right]^{j-n} \\
& \times\left[\exp _{p}\left\{(\beta+\varepsilon) \log _{q} r\right\}\right]^{n-j} \\
= & {\left[\frac{r \exp _{p}\left\{(\beta+\varepsilon) \log _{q} r\right\}}{\exp _{p}\left\{(\mu-\varepsilon) \log _{q} r\right\}}\right]^{n-j} \rightarrow 0 }
\end{aligned}
$$

as $r \rightarrow+\infty$, where $|z|=r \notin[0,1] \cup E_{5}, E_{5}=E_{1} \cup E_{4}$ and $|g(z)|=M(r, g)$. From (2.17) and (2.19), we obtain our assertion. 
Lemma 2.8 Let $f(z)=\frac{g(z)}{d(z)}$ be a meromorphic function, where $g(z), d(z)$ are entire functions satisfying $\mu_{[p, q]}(g)=\mu_{[p, q]}(f)=\mu \leq \rho_{[p, q]}(f)=\rho_{[p, q]}(g) \leq+\infty$ and $\lambda_{[p, q]}(d)=\rho_{[p, q]}(d)=$ $\lambda_{[p, q]}\left(\frac{1}{f}\right)<\mu$. Then, there exists a set $E_{6} \subset(1,+\infty)$ of finite logarithmic measure such that for all $|z|=r \notin[0,1] \cup E_{6}$ and $|g(z)|=M(r, g)$, we have

$$
\left|\frac{f(z)}{f(s)(z)}\right| \leq r^{2 s}, \quad(s \in \mathbb{N}) .
$$

Proof. By Lemma 2.7, there exists a set $E_{5}$ of finite logarithmic measure such that the estimation

$$
\frac{f^{(s)}(z)}{f(z)}=\left(\frac{\nu_{g}(r)}{z}\right)^{s}(1+o(1)) \quad(s \geq 1 \text { is an integer })
$$

holds for all $|z|=r \notin[0,1] \cup E_{5}$ and $|g(z)|=M(r, g)$, where $\nu_{g}(r)$ is the central index of $g$. On the other hand, by Lemma 2.6, for any given $\varepsilon(0<\varepsilon<1)$, there exists $R>1$ such that for all $r>R$, we have

$$
\nu_{g}(r)>\exp _{p}\left\{(\mu-\varepsilon) \log _{q}(r)\right\}
$$

If $\mu=+\infty$, then $\mu-\varepsilon$ can be replaced by a large enough real number $M$. Set $E_{6}=[1, R] \cup E_{5}$, $\operatorname{Im}\left(E_{6}\right)<+\infty$. Hence from (2.20) and (2.21), we obtain

$$
\left|\frac{f(z)}{f(s)(z)}\right|=\left|\frac{z}{\nu_{g}(r)}\right|^{s} \frac{1}{|1+o(1)|} \leq \frac{r^{s}}{\left(\exp _{p}\left\{(\mu-\varepsilon) \log _{q}(r)\right\}\right)^{s}} \leq r^{2 s},
$$

where $|z|=r \notin[0,1] \cup E_{6}, r \rightarrow+\infty$ and $|g(z)|=M(r, g)$.

Lemma $2.9([6])$ Let $\varphi:[0,+\infty) \rightarrow \mathbb{R}$ and $\psi:[0,+\infty) \rightarrow \mathbb{R}$ be monotone nondecreasing functions such that $\varphi(r) \leq \psi(r)$ for all $r \notin\left(E_{7} \cup[0,1]\right)$, where $E_{7}$ is a set of finite logarithmic measure. Let $\alpha>1$ be a given constant. Then, there exists an $r_{1}=r_{1}(\alpha)>0$ such that $\varphi(r) \leq \psi(\alpha r)$ for all $r>r_{1}$.

Lemma 2.10 ([19]) Let $f(z)=\frac{g(z)}{d(z)}$ be a meromorphic function, where $g(z), d(z)$ are entire functions. If $0 \leq \rho_{[p, q]}(d)<\mu_{[p, q]}(f)$, then $\mu_{[p, q]}(g)=\mu_{[p, q]}(f)$ and $\rho_{[p, q]}(g)=\rho_{[p, q]}(f)$. Moreover, if $\rho_{[p, q]}(f)=+\infty$, then $\rho_{[p+1, q]}(g)=\rho_{[p+1, q]}(f)$.

Lemma 2.11 Assume that $k \geq 2$ and $A_{0}, A_{1}, \ldots, A_{k} \not \equiv 0, F$ are meromorphic functions. Let $\rho=\max \left\{\rho_{[p, q]}\left(A_{j}\right)(j=0,1, \ldots, k), \rho_{[p, q]}(F)\right\}<\infty$ and let $f$ be a meromorphic solution of infinite $[p, q]$-order of equation (1.4) with $\lambda_{[p, q]}\left(\frac{1}{f}\right)<\mu_{[p, q]}(f)$. Then, $\rho_{[p+1, q]}(f) \leq \rho$.

Proof. Let $f$ be a meromorphic solution of infinite $[p, q]$-order of equation (1.4) with $\lambda_{[p, q]}\left(\frac{1}{f}\right)<$ $\mu_{[p, q]}(f)$. So, we can use Hadamard factorization theorem and write $f$ as $f(z)=\frac{g(z)}{d(z)}$, where $g(z)$ and $d(z)$ are entire functions satisfying $\mu_{[p, q]}(g)=\mu_{[p, q]}(f)=\mu \leq \rho_{[p, q]}(f)=\rho_{[p, q]}(g) \leq+\infty$ 
and $\lambda_{[p, q]}(d)=\rho_{[p, q]}(d)=\lambda_{[p, q]}\left(\frac{1}{f}\right)<\mu$. By Lemma 2.3, there exists a set $E_{3} \subset(1,+\infty)$ of $r$ with a finite linear measure such that for all $|z|=r \notin E_{3}$ and any given $\varepsilon(0<2 \varepsilon<$ $\left.\mu_{[p, q]}(f)-\rho_{[p, q]}(d)\right)$, we have

$$
\begin{gathered}
\left|A_{j}(z)\right| \leq \exp _{p+1}\left\{\left(\rho_{(p, q)}\left(A_{j}\right)+\varepsilon\right) \log _{q} r\right\} \\
\leq \exp _{p+1}\left\{(\rho+\varepsilon) \log _{q} r\right\}, j=0,1, \ldots, k-1, \\
\left|A_{k}(z)\right| \geq \exp \left\{-\exp _{p}\left\{\left(\rho_{(p, q)}\left(A_{k}\right)+\varepsilon\right) \log _{q} r\right\}\right\} \\
\geq \exp \left\{-\exp _{p}\left\{(\rho+\varepsilon) \log _{q} r\right\}\right\}
\end{gathered}
$$

and

$$
|F(z)| \leq \exp _{p+1}\left\{\left(\rho_{(p, q)}(F)+\varepsilon\right) \log _{q} r\right\} \leq \exp _{p+1}\left\{(\rho+\varepsilon) \log _{q} r\right\} .
$$

By (2.24), for all $z$ satisfying $|z|=r \notin E_{3}$ at which $|g(z)|=M(r, g)$ and any given $\varepsilon\left(0<2 \varepsilon<\mu_{[p, q]}(f)-\rho_{[p, q]}(d)\right)$, we obtain

$$
\begin{gathered}
\left|\frac{F(z)}{f(z)}\right|=\frac{|F(z)|}{|g(z)|}|d(z)| \\
\leq \frac{\exp _{p+1}\left\{\left(\rho_{[p, q]}(d)+\varepsilon\right) \log _{q} r\right\} \exp _{p+1}\left\{(\rho+\varepsilon) \log _{q} r\right\}}{\exp _{p+1}\left\{\left(\mu_{[p, q]}(f)-\varepsilon\right) \log _{q} r\right\}} \\
\leq \exp _{p+1}\left\{(\rho+\varepsilon) \log _{q} r\right\} .
\end{gathered}
$$

By Lemma 2.7, there exists a set $E_{5} \subset(1,+\infty)$ of finite logarithmic measure such that for all $|z|=r \notin[0,1] \cup E_{5}$ and $|g(z)|=M(r, g)$, we have

$$
\frac{f^{(j)}(z)}{f(z)}=\left(\frac{\nu_{g}(r)}{z}\right)^{j}(1+o(1)), j=1, \ldots, k .
$$

We can rewrite (1.4) as

$$
\left|\frac{f^{(k)}(z)}{f(z)}\right| \leq \frac{1}{\left|A_{k}(z)\right|}\left(\left|A_{0}(z)\right|+\left|\frac{F(z)}{f(z)}\right|+\sum_{j=1}^{k-1}\left|A_{j}(z)\right| \frac{f^{(j)}(z)}{f(z)} \mid\right) .
$$

By substituting (2.22), (2.23), (2.25) and (2.26) into (2.27), we obtain

$$
\begin{gathered}
\left|\frac{\nu_{g}(r)}{z}\right|^{k}|1+o(1)| \leq \frac{1}{\exp \left\{-\exp _{p}\left\{(\rho+\varepsilon) \log _{q} r\right\}\right\}} \times \\
\left(\left\{1+\sum_{j=1}^{k-1}\left|\frac{\nu_{g}(r)}{z}\right|^{j}|1+o(1)|\right\} \exp _{p+1}\left\{(\rho+\varepsilon) \log _{q} r\right\}\right. \\
\left.+\exp _{p+1}\left\{(\rho+\varepsilon) \log _{q} r\right\}\right) \\
=\left\{2+\sum_{j=1}^{k-1}\left|\frac{\nu_{g}(r)}{z}\right|^{j}|1+o(1)|\right\} \exp \left\{2 \exp _{p}\left\{(\rho+\varepsilon) \log _{q} r\right\}\right\} .
\end{gathered}
$$

Hence

$$
\left|\nu_{g}(r)\right||1+o(1)| \leq(k+1) r|1+o(1)| \exp \left\{2 \exp _{p}\left\{(\rho+\varepsilon) \log _{q} r\right\}\right\}
$$


holds for all $z$ satisfying $|z|=r \notin[0,1] \cup E_{3} \cup E_{5}$ and $|g(z)|=M(r, g), r \rightarrow+\infty$. By (2.28), we get

$$
\limsup _{r \rightarrow+\infty} \frac{\log _{p+1} \nu_{g}(r)}{\log _{q} r} \leq \rho+\varepsilon .
$$

Since $\varepsilon>0$ is arbitrary, by (2.29) and Lemma 2.6, we obtain $\rho_{[p+1, q]}(g) \leq \rho$. Since $\rho_{[p, q]}(d)<$ $\mu_{[p, q]}(f)$, so by Lemma 2.10, we have $\rho_{[p+1, q]}(g)=\rho_{[p+1, q]}(f)$. Thus, $\rho_{[p+1, q]}(f) \leq \rho$. Therefore, Lemma 2.11 is proved.

Lemma $2.12([19])$ Let $A_{j}(z)(j=0,1, \ldots, k), A_{k}(z)(\not \equiv 0), F(z)(\not \equiv 0)$ be meromorphic functions and let $f$ be a meromorphic solution of (1.4) of infinite $[p, q]$-order satisfying the following condition

$$
b=\max \left\{\rho_{[p+1, q]}(F), \rho_{[p+1, q]}\left(A_{j}\right)(j=0,1, \ldots, k)\right\}<\rho_{[p+1, q]}(f) .
$$

Then

$$
\bar{\lambda}_{[p+1, q]}(f)=\lambda_{[p+1, q]}(f)=\rho_{[p+1, q]}(f) .
$$

Lemma 2.13 Let $H \subset(1,+\infty)$ be a set with a positive upper logarithmic density (or infinite logarithmic measure), and let $A_{j}(z)(j=0,1, \ldots, k)$ with $A_{k}(z) \not \equiv 0$ and $F(z) \not \equiv 0$ be meromorphic functions with finite $[p, q]$-order. If there exist a positive constant $\sigma>0$ and an integer $s$, $0 \leq s \leq k$, such that for sufficiently small $\varepsilon>0$, we have $\left|A_{s}(z)\right| \geq \exp _{p+1}\left\{(\sigma-\varepsilon) \log _{q} r\right\}$ as $|z|=r \in H, r \rightarrow+\infty$ and

$$
\max \left\{\rho_{[p, q]}\left(A_{j}\right)(j \neq s), \rho_{[p, q]}(F)\right\}<\sigma,
$$

then every transcendental meromorphic solution $f$ of equation (1.4) satisfies $\rho_{[p, q]}(f) \geq \sigma$.

Proof. Assume that $f$ is a transcendental meromorphic solution of equation $(1.4)$ with $\rho_{[p, q]}(f)<\sigma$. From (1.4), we have

$$
A_{s}=\frac{F}{f(s)}-\sum_{\substack{j=0 \\ j \neq s}}^{k} A_{j} \frac{f^{(j)}}{f(s)} .
$$

Since $\max \left\{\rho_{[p, q]}\left(A_{j}\right)(j \neq s), \rho_{[p, q]}(F)\right\}<\sigma$ and $\rho_{[p, q]}(f)<\sigma$, then from (2.30) we obtain that

$$
\rho_{1}=\rho_{[p, q]}\left(A_{s}\right) \leq \max \left\{\rho_{[p, q]}\left(A_{j}\right)(j \neq s), \rho_{[p, q]}(F), \rho_{[p, q]}(f)\right\}<\sigma .
$$

By Lemma 2.3, for any $\varepsilon\left(0<2 \varepsilon<\sigma-\rho_{1}\right)$, there exists a set $E_{3} \subset(1,+\infty)$ with a finite linear measure such that

$$
\left|A_{s}(z)\right| \leq \exp _{p+1}\left\{\left(\rho_{(p, q)}\left(A_{s}\right)+\varepsilon\right) \log _{q} r\right\}=\exp _{p+1}\left\{\left(\rho_{1}+\varepsilon\right) \log _{q} r\right\}
$$

holds for all $z$ satisfying $|z|=r \notin E_{3}$. From the hypotheses of Lemma 2.13, there exists a set $H$ with $\overline{\log d e n s} H>0$ (or $\left.m_{l}(H)=+\infty\right)$ such that

$$
\left|A_{s}(z)\right| \geq \exp _{p+1}\left\{(\sigma-\varepsilon) \log _{q} r\right\}
$$


holds for all $z$ satisfying $|z|=r \in H, r \rightarrow+\infty$. By (2.31) and (2.32), we conclude that for all $z$ satisfying $|z|=r \in H \backslash E_{3}, r \rightarrow+\infty$, we have

$$
\exp _{p+1}\left\{(\sigma-\varepsilon) \log _{q} r\right\} \leq \exp _{p+1}\left\{\left(\rho_{1}+\varepsilon\right) \log _{q} r\right\}
$$

and by $\varepsilon\left(0<2 \varepsilon<\sigma-\rho_{1}\right)$ this is a contradiction as $r \rightarrow+\infty$. Consequently, any transcendental meromorphic solution $f$ of equation (1.4) satisfies $\rho_{[p, q]}(f) \geq \sigma$.

Lemma 2.14 ([23]) Let $p \geq q \geq 1$ be integers. Let $f$ be a meromorphic function for which $\rho_{[p, q]}(f)=\beta<+\infty$, and let $k \geq 1$ be an integer. Then for any $\varepsilon>0$,

$$
m\left(r, \frac{f^{(k)}}{f}\right)=O\left(\exp _{p-1}\left\{(\beta+\varepsilon) \log _{q} r\right\}\right),
$$

holds outside of a possible exceptional set $E_{8}$ of finite linear measure.

Lemma 2.15 Let $A_{0}, A_{1}, \ldots, A_{k} \not \equiv 0, F \not \equiv 0$ be finite $[p, q]$-order meromorphic functions. If $f$ is $a$ meromorphic solution with $\rho_{[p, q]}(f)=+\infty$ and $\rho_{[p+1, q]}(f)=\rho<+\infty$ of equation $(1.4)$, then $\bar{\lambda}_{[p, q]}(f)=\lambda_{[p, q]}(f)=\rho_{[p, q]}(f)=+\infty$ and $\bar{\lambda}_{[p+1, q]}(f)=\lambda_{[p+1, q]}(f)=\rho_{[p+1, q]}(f)=\rho$.

Proof Let $f$ be a meromorphic solution of (1.4) with infinite $[p, q]$-order and $\rho_{[p+1, q]}(f)=$ $\rho<+\infty$. Note first that by definition, we have $\bar{\lambda}_{[p+1, q]}(f) \leq \lambda_{[p+1, q]}(f) \leq \rho_{[p+1, q]}(f)$. Then, it remains to show that

$$
\rho_{[p+1, q]}(f) \leq \bar{\lambda}_{[p+1, q]}(f) \leq \lambda_{[p+1, q]}(f)
$$

We rewrite (1.4) as

$$
\frac{1}{f}=\frac{1}{F}\left(A_{k}(z) \frac{f^{(k)}}{f}+A_{k-1}(z) \frac{f^{(k-1)}}{f}+\cdots+A_{1}(z) \frac{f^{\prime}}{f}+A_{0}(z)\right) .
$$

By using Lemma 2.14 and (2.33), for $|z|=r$ outside a set $E_{8}$ of a finite linear measure and any given $\varepsilon>0$, we get

$$
\begin{gathered}
m\left(r, \frac{1}{f}\right) \leq m\left(r, \frac{1}{F}\right)+\sum_{j=1}^{k} m\left(r, \frac{f^{(j)}}{f}\right)+\sum_{j=0}^{k} m\left(r, A_{j}\right)+O(1) \\
\leq m\left(r, \frac{1}{F}\right)+\sum_{j=0}^{k} m\left(r, A_{j}\right)+O\left(\exp _{p}\left\{(\rho+\varepsilon) \log _{q} r\right\}\right) .
\end{gathered}
$$

On the other hand, by (1.4), if $f$ has a zero at $z_{0}$ of order $\alpha(\alpha>k)$, and $A_{0}, A_{1}, \ldots, A_{k}$ are all analytic at $z_{0}$, then $F$ must have a zero at $z_{0}$ of order at least $\alpha-k$. Hence,

$$
n\left(r, \frac{1}{f}\right) \leq k \bar{n}\left(r, \frac{1}{f}\right)+n\left(r, \frac{1}{F}\right)+\sum_{j=0}^{k} n\left(r, A_{j}\right)
$$


and

$$
N\left(r, \frac{1}{f}\right) \leq k \bar{N}\left(r, \frac{1}{f}\right)+N\left(r, \frac{1}{F}\right)+\sum_{j=0}^{k} N\left(r, A_{j}\right) .
$$

Therefore, by (2.34) and (2.35), for all sufficiently large $r \notin E_{8}$ and any given $\varepsilon>0$, we have

$$
\begin{aligned}
T(r, f) & =T\left(r, \frac{1}{f}\right)+O(1) \leq T(r, F)+\sum_{j=0}^{k} T\left(r, A_{j}\right) \\
& +k \bar{N}\left(r, \frac{1}{f}\right)+O\left(\exp _{p}\left\{(\rho+\varepsilon) \log _{q} r\right\}\right) .
\end{aligned}
$$

Noting $c=\max \left\{\rho_{[p, q]}\left(A_{j}\right)(j=0,1, \ldots, k), \rho_{[p, q]}(F)\right\}$. Then, by using the definition of the $[p, q]-$ order, for the above $\varepsilon$ and sufficiently large $r$, we have

$$
\begin{gathered}
T(r, F) \leq \exp _{p}\left\{(c+\varepsilon) \log _{q} r\right\}, \\
T\left(r, A_{j}\right) \leq \exp _{p}\left\{(c+\varepsilon) \log _{q} r\right\}, j=0,1, \ldots, k .
\end{gathered}
$$

Replacing (2.37) and (2.38) into (2.36), for $r \notin E_{8}$ sufficiently large and any given $\varepsilon>0$, we obtain

$$
T(r, f) \leq k \bar{N}\left(r, \frac{1}{f}\right)+(k+2) \exp _{p}\left\{(c+\varepsilon) \log _{q} r\right\}+O\left(\exp _{p}\left\{(\rho+\varepsilon) \log _{q} r\right\}\right) .
$$

Hence, for any $f$ with $\rho_{[p, q]}(f)=+\infty$ and $\rho_{[p+1, q]}(f)=\rho$, by (2.39), we have

$$
\bar{\lambda}_{[p, q]}(f) \geq \rho_{[p, q]}(f)=+\infty, \bar{\lambda}_{[p+1, q]}(f) \geq \rho_{[p+1, q]}(f),
$$

so

$$
\rho_{[p+1, q]}(f) \leq \bar{\lambda}_{[p+1, q]}(f) \leq \lambda_{[p+1, q]}(f) .
$$

And the fact that $\bar{\lambda}_{[p+1, q]}(f) \leq \lambda_{[p+1, q]}(f) \leq \rho_{[p+1, q]}(f)$, we obtain

$$
\bar{\lambda}_{[p+1, q]}(f)=\lambda_{[p+1, q]}(f)=\rho_{[p+1, q]}(f)=\rho .
$$

\section{Proof of Theorem 1.1}

Assume that $f \not \equiv 0$ is a rational solution of (1.3). First, we will prove that $f$ must be a polynomial with $\operatorname{deg} f \leq s-1$. For this, if $f$ is a rational function, which has a pole at $z_{0}$ of degree $m \geq 1$, or $f$ is a polynomial with $\operatorname{deg} f \geq s$, then $f^{(s)}(z) \not \equiv 0$. By (1.3) and Lemma 2.4, we obtain

$$
\begin{aligned}
\sigma \leq \rho_{[p, q]}\left(A_{s}\right)= & \rho_{[p, q]}\left(A_{s} f^{(s)}\right)=\rho_{[p, q]}\left(-\left(\sum_{j=0, j \neq s}^{k} A_{j} f^{(j)}\right)\right) \\
& \leq \max _{j=0,1, \ldots, k, j \neq s}\left\{\rho_{[p, q]}\left(A_{j}\right)\right\}
\end{aligned}
$$

which is a contradiction. Therefore, $f$ must be a polynomial with $\operatorname{deg} f \leq s-1$. 
Now, we assume that $f$ is a transcendental meromorphic solution of $(1.3)$ such that $\lambda_{[p, q]}\left(\frac{1}{f}\right)<$ $\mu_{[p, q]}(f)$. By Lemma 2.3, for any given $\varepsilon(0<2 \varepsilon<\sigma-\rho)$, there exists a set $E_{3} \subset(1,+\infty)$ with a finite linear measure (and so of finite logarithmic measure) such that

$$
\left|A_{j}(z)\right| \leq \exp _{p+1}\left\{(\rho+\varepsilon) \log _{q} r\right\}, j=0,1, \ldots, k, j \neq s
$$

holds for all $z$ satisfying $|z|=r \notin E_{3}$. In view of Lemma 2.8, there exists a set $E_{6} \subset(1,+\infty)$ of finite logarithmic measure such that $|z|=r \notin[0,1] \cup E_{6},|g(z)|=M(r, g)$ and for $r$ sufficiently large, we have

$$
\left|\frac{f(z)}{f(s)(z)}\right| \leq r^{2 s} \quad(s \geq 1 \text { is an integer }) .
$$

According to Lemma 2.1, there exist a set $E_{1} \subset(1,+\infty)$ with $m_{l}\left(E_{1}\right)<\infty$ and a constant $B>0$, such that for all $z$ satisfying $|z|=r \notin[0,1] \cup E_{1}$, we have

$$
\left|\frac{f^{(j)}(z)}{f(z)}\right| \leq B[T(2 r, f)]^{k+1}, j=1,2, \ldots, k, j \neq s .
$$

From the hypotheses of Theorem 1.1, there exists a set $H \subset(1,+\infty)$ with $m_{l}(H)=+\infty$, such that for all $z$ satisfying $|z|=r \in H, r \rightarrow+\infty$ and sufficiently small $\varepsilon>0$, we have

$$
\left|A_{s}(z)\right| \geq \exp _{p+1}\left\{(\sigma-\varepsilon) \log _{q} r\right\} .
$$

Now, by rewriting equation (1.3) in the form

$$
\left|A_{s}\right| \leq\left|\frac{f}{f(s)}\right|\left(\left|A_{0}\right|+\sum_{\substack{j=1 \\ j \neq s}}^{k}\left|A_{j}\right|\left|\frac{f^{(j)}}{f}\right|\right)
$$

and substituting (3.1), (3.2), (3.3) and (3.4) into (3.5), for all $z$ satisfying $|z|=r \in H \backslash([0,1] \cup$ $\left.E_{1} \cup E_{3} \cup E_{6}\right), r \rightarrow+\infty$, we obtain

$$
\exp _{p+1}\left\{(\sigma-\varepsilon) \log _{q} r\right\} \leq B k r^{2 s} \exp _{p+1}\left\{(\rho+\varepsilon) \log _{q} r\right\}[T(2 r, f)]^{k+1} .
$$

Since $0<2 \varepsilon<\sigma-\rho$, then we have

$$
\exp \left\{(1-o(1)) \exp _{p}\left\{(\sigma-\varepsilon) \log _{q} r\right\}\right\} \leq B k r^{2 s}[T(2 r, f)]^{k+1}
$$

From (3.6) and Lemma 2.9, for any given $\gamma>1$ and sufficiently large $r>R$, we get

$$
\exp \left\{(1-o(1)) \exp _{p}\left\{(\sigma-\varepsilon) \log _{q} r\right\}\right\} \leq B k(\gamma r)^{2 s}[T(2 \gamma r, f)]^{k+1}
$$

which gives

$$
\rho_{[p, q]}(f)=\mu_{[p, q]}(f)=+\infty, \sigma \leq \rho_{[p+1, q]}(f) .
$$

By using Lemma 2.4, we have

$$
\max \left\{\rho_{[p, q]}\left(A_{j}\right): j=0,1, \ldots, k\right\}=\rho_{[p, q]}\left(A_{s}\right)=\beta<+\infty .
$$


Since $f$ is of infinite $[p, q]$-order meromorphic solution of equation (1.3) satisfying $\lambda_{[p, q]}\left(\frac{1}{f}\right)<$ $\mu_{[p, q]}(f)$, then by Lemma 2.11, we obtain

$$
\rho_{[p+1, q]}(f) \leq \max \left\{\rho_{[p, q]}\left(A_{j}\right): j=0,1, \ldots, k\right\}=\rho_{[p, q]}\left(A_{s}\right) .
$$

By (3.7) and (3.8), we conclude that $\mu_{[p, q]}(f)=\rho_{[p, q]}(f)=+\infty$ and $\sigma \leq \rho_{[p+1, q]}(f) \leq$ $\rho_{[p, q]}\left(A_{S}\right)$.

\section{Proof of Corollary 1.1}

Assume that $\varphi$ is a transcendental meromorphic function such that $\rho_{[p+1, q]}(\varphi)<\sigma$. Noting $g=$ $f-\varphi$, then $\rho_{[p+1, q]}(g)=\rho_{[p+1, q]}(f)$, so by Theorem 1.1, $\sigma \leq \rho_{[p+1, q]}(g) \leq \rho_{[p, q]}\left(A_{s}\right)$. By substituting $f=g+\varphi$ into (1.3), we obtain

$$
\begin{gathered}
A_{k}(z) g^{(k)}+A_{k-1}(z) g^{(k-1)}+\cdots+A_{1}(z) g^{\prime}+A_{0}(z) g \\
=-\left(A_{k}(z) \varphi^{(k)}+A_{k-1}(z) \varphi^{(k-1)}+\cdots+A_{1}(z) \varphi^{\prime}+A_{0}(z) \varphi\right)=G(z) .
\end{gathered}
$$

It is clear that the right side $G$ of equation (4.1) is non-zero, because by Theorem 1.1, $\varphi$ is not a solution of equation (1.3). Moreover, the $[p+1, q]$-order of $G$ satisfies

$$
\rho_{[p+1, q]}(G) \leq \max \left\{\rho_{[p+1, q]}(\varphi), \rho_{[p+1, q]}\left(A_{j}\right)(j=0,1, \ldots, k)\right\}<\sigma,
$$

which implies

$$
\max \left\{\rho_{[p+1, q]}(G), \rho_{[p+1, q]}\left(A_{j}\right) \quad(j=0,1, \ldots, k)\right\}<\sigma \leq \rho_{[p+1, q]}(g) .
$$

Then by Lemma 2.12, we obtain

$$
\begin{gathered}
\sigma \leq \bar{\lambda}_{[p+1, q]}(g)=\lambda_{[p+1, q]}(g) \\
=\rho_{[p+1, q]}(g)=\rho_{[p+1, q]}(f) \leq \rho_{[p, q]}\left(A_{s}\right),
\end{gathered}
$$

that is

$$
\begin{gathered}
\sigma \leq \bar{\lambda}_{[p+1, q]}(f-\varphi)=\lambda_{[p+1, q]}(f-\varphi) \\
=\rho_{[p+1, q]}(f-\varphi)=\rho_{[p+1, q]}(f) \leq \rho_{[p, q]}\left(A_{s}\right) .
\end{gathered}
$$

\section{Proof of Theorem 1.2}

Assume that $f$ is a rational solution of (1.4). First, we will prove that $f$ must be a polynomial with $\operatorname{deg} f \leq s-1$. For this, if $f$ is a rational function, which has a pole at $z_{0}$ of degree $m \geq 1$, or $f$ is a polynomial with $\operatorname{deg} f \geq s$, then $f^{(s)}(z) \not \equiv 0$. By (1.4) and Lemma 2.4, we obtain

$$
\begin{gathered}
\sigma \leq \rho_{[p, q]}\left(A_{s}\right)=\rho_{[p, q]}\left(A_{s} f^{(s)}\right)=\rho_{[p, q]}\left(F-\sum_{\substack{j=0 \\
j \neq s}}^{k} A_{j}(z) f^{(j)}\right) \\
\leq \max _{j=0,1, \ldots, k, j \neq s}\left\{\rho_{[p, q]}\left(A_{j}\right), \rho_{[p, q]}(F)\right\},
\end{gathered}
$$


which is a contradiction. Therefore, $f$ must be a polynomial with $\operatorname{deg} f \leq s-1$.

Now, we assume that $f$ is a transcendental meromorphic solution of $(1.4)$ such that $\lambda_{[p, q]}\left(\frac{1}{f}\right)<$ $\mu_{[p, q]}(f)$. From Lemma 2.13, we know that $f$ satisfies $\rho_{[p, q]}(f) \geq \sigma$. By the hypothesis $\lambda_{[p, q]}\left(\frac{1}{f}\right)<$ $\min \left\{\mu_{[p, q]}(f), \sigma\right\}$ and Hadamard factorization theorem, we can write $f$ as $f(z)=\frac{g(z)}{d(z)}$, where $g(z)$ and $d(z)$ are entire functions satisfying

$$
\begin{gathered}
\mu_{[p, q]}(g)=\mu_{[p, q]}(f)=\mu \leq \rho_{[p, q]}(g)=\rho_{[p, q]}(f), \\
\rho_{[p, q]}(d)=\lambda_{[p, q]}\left(\frac{1}{f}\right)=\beta<\min \left\{\mu_{[p, q]}(f), \sigma\right\} .
\end{gathered}
$$

The definition of the lower $[p, q]$-order assures us that

$$
|g(z)|=M(r, g) \geq \exp _{p+1}\left\{\left(\mu_{[p, q]}(g)-\varepsilon\right) \log _{q} r\right\} .
$$

Putting

$$
\rho_{1}=\max \left\{\rho_{[p, q]}\left(A_{j}\right)(j \neq s), \rho_{[p, q]}(F)\right\}<\sigma .
$$

Then, by Lemma 2.3 and (5.1), for any given $\varepsilon$ satisfying

$$
0<2 \varepsilon<\min \left\{\sigma-\rho_{1}, \mu_{[p, q]}(g)-\rho_{[p, q]}(d)\right\},
$$

there exists a set $E_{3} \subset(1,+\infty)$ with a finite logarithmic measure such that for all $z$ satisfying $|z|=r \notin E_{3}$ at which $|g(z)|=M(r, g)$, we obtain

$$
\begin{gathered}
\left|\frac{F(z)}{f(z)}\right|=\frac{|F(z)|}{|g(z)|}|d(z)| \\
\leq \frac{\exp _{p+1}\left\{\left(\rho_{[p, q]}(d)+\varepsilon\right) \log _{q} r\right\} \exp _{p+1}\left\{\left(\rho_{1}+\varepsilon\right) \log _{q} r\right\}}{\exp _{p+1}\left\{\left(\mu_{[p, q]}(g)-\varepsilon\right) \log _{q} r\right\}} \\
\leq \exp _{p+1}\left\{\left(\rho_{1}+\varepsilon\right) \log _{q} r\right\} .
\end{gathered}
$$

By using the same arguments as in the proof of Theorem 1.1, for any given $\varepsilon\left(0<2 \varepsilon<\min \left\{\sigma-\rho_{1}, \mu_{[p, q]}(g)-\rho_{[p, q]}(d)\right\}\right)$ and all $z$ satisfying $|z|=r \in H \backslash\left(E_{1} \cup E_{3} \cup E_{6}\right)$, $r \rightarrow+\infty$ at which $|g(z)|=M(r, g)$, we have (3.2), (3.3), (3.4) hold and

$$
\left|A_{j}(z)\right| \leq \exp _{p+1}\left\{\left(\rho_{1}+\varepsilon\right) \log _{q} r\right\}, j=0,1, \ldots, k, j \neq s .
$$

By (1.4), we have

$$
\left|A_{s}\right| \leq\left|\frac{f}{f^{(s)}}\right|\left(\left|A_{0}\right|+\sum_{\substack{j=1 \\ j \neq s}}^{k}\left|A_{j}\right|\left|\frac{f^{(j)}}{f}\right|+\left|\frac{F}{f}\right|\right) .
$$

Hence, by substituting (3.2), (3.3) , (3.4), (5.2) and (5.3) into (5.4), for all $z$ satisfying $|z|=r \in$ $H \backslash\left(E_{1} \cup E_{3} \cup E_{6}\right), r \rightarrow+\infty$, at which $|g(z)|=M(r, g)$ and any given $\varepsilon\left(0<2 \varepsilon<\min \left\{\sigma-\rho_{1}, \mu_{[p, q]}(g)-\rho_{[p, q]}(d)\right\}\right)$, we obtain

$$
\exp _{p+1}\left\{(\sigma-\varepsilon) \log _{q} r\right\} \leq r^{2 s}\left(\exp _{p+1}\left\{\left(\rho_{1}+\varepsilon\right) \log _{q} r\right\}\right.
$$




$$
\begin{gathered}
+\sum_{j=1, j \neq s}^{k} \exp _{p+1}\left\{\left(\rho_{1}+\varepsilon\right) \log _{q} r\right\} B[T(2 r, f)]^{k+1} \\
\left.+\exp _{p+1}\left\{\left(\rho_{1}+\varepsilon\right) \log _{q} r\right\}\right) \\
\leq B(k+1) r^{2 s}[T(2 r, f)]^{k+1} \exp _{p+1}\left\{\left(\rho_{1}+\varepsilon\right) \log _{q} r\right\} .
\end{gathered}
$$

Since $0<2 \varepsilon<\sigma-\rho_{1}$, then we can use Lemma 2.9 with (5.5) such that for any given $\gamma>1$ and sufficiently large $r>R$, we obtain

$$
\exp \left\{(1-o(1)) \exp _{p}\left\{(\sigma-\varepsilon) \log _{q} r\right\}\right\} \leq B(k+1)(\gamma r)^{2 s}[T(2 \gamma r, f)]^{k+1}
$$

which gives

$$
\rho_{[p, q]}(f)=\mu_{[p, q]}(f)=+\infty, \rho_{[p+1, q]}(f) \geq \sigma .
$$

Making use of Lemma 2.4, we have

$$
\max \left\{\rho_{[p, q]}\left(A_{j}\right)(j=0,1, \ldots, k), \rho_{[p, q]}(F)\right\}=\rho_{[p, q]}\left(A_{s}\right)=\beta<+\infty .
$$

By Lemma 2.11 and since $f$ is of infinite $[p, q]$-order meromorphic solution of equation (1.4) satisfying $\lambda_{[p, q]}\left(\frac{1}{f}\right)<\mu_{[p, q]}(f)$, we get

$$
\rho_{[p+1, q]}(f) \leq \max \left\{\rho_{[p, q]}\left(A_{j}\right) \quad(j=0,1, \ldots, k), \rho_{[p, q]}(F)\right\}=\rho_{[p, q]}\left(A_{s}\right) .
$$

Since $F \not \equiv 0$, then by Lemma 2.15 , we have

$$
\bar{\lambda}_{[p, q]}(f)=\lambda_{[p, q]}(f)=\mu_{[p, q]}(f)=\rho_{[p, q]}(f)=+\infty
$$

and

$$
\sigma \leq \bar{\lambda}_{[p+1, q]}(f)=\lambda_{[p+1, q]}(f)=\rho_{[p+1, q]}(f) .
$$

By (5.7), (5.8) and (5.9), we conclude that

$$
\bar{\lambda}_{[p, q]}(f)=\lambda_{[p, q]}(f)=\mu_{[p, q]}(f)=\rho_{[p, q]}(f)=+\infty
$$

and

$$
\sigma \leq \bar{\lambda}_{[p+1, q]}(f)=\lambda_{[p+1, q]}(f)=\rho_{[p+1, q]}(f) \leq \rho_{[p, q]}\left(A_{s}\right)
$$

\section{Proof of Corollary 1.2}

Assume that $\varphi$ is a transcendental meromorphic function such that $\rho_{[p+1, q]}(\varphi)<\sigma$. Noting $h=$ $f-\varphi$, then $\rho_{[p+1, q]}(h)=\rho_{[p+1, q]}(f)$, so by Theorem 1.2, $\sigma \leq \rho_{[p+1, q]}(h) \leq \rho_{[p, q]}\left(A_{s}\right)$. By substituting $f=h+\varphi$ into (1.4), we obtain

$$
\begin{gathered}
A_{k}(z) h^{(k)}+A_{k-1}(z) h^{(k-1)}+\cdots+A_{1}(z) h^{\prime}+A_{0}(z) h \\
=F(z)-\left(A_{k}(z) \varphi^{(k)}+A_{k-1}(z) \varphi^{(k-1)}+\cdots+A_{1}(z) \varphi^{\prime}+A_{0}(z) \varphi\right)=\Psi(z) .
\end{gathered}
$$


It is clear that the right side $\Psi$ of the equation (6.1) is non-zero, because by Theorem $1.2, \varphi$ is not a solution of equation (1.4). Moreover, the $[p+1, q]$-order of $\psi$ verifies

$$
\rho_{[p+1, q]}(\Psi) \leq \max \left\{\rho_{[p+1, q]}(\varphi), \rho_{[p+1, q]}\left(A_{j}\right)(j=0,1, \ldots, k)\right\}<\sigma
$$

which leads to

$$
\max \left\{\rho_{[p+1, q]}(\Psi), \rho_{[p+1, q]}\left(A_{j}\right) \quad(j=0,1, \ldots, k)\right\}<\sigma \leq \rho_{[p+1, q]}(h) .
$$

Therefore, by Lemma 2.12, we obtain

$$
\begin{gathered}
\sigma \leq \bar{\lambda}_{[p+1, q]}(h)=\lambda_{[p+1, q]}(h) \\
=\rho_{[p+1, q]}(h)=\rho_{[p+1, q]}(f) \leq \rho_{[p, q]}\left(A_{s}\right),
\end{gathered}
$$

that is

$$
\begin{gathered}
\sigma \leq \bar{\lambda}_{[p+1, q]}(f-\varphi)=\lambda_{[p+1, q]}(f-\varphi) \\
=\rho_{[p+1, q]}(f-\varphi)=\rho_{[p+1, q]}(f) \leq \rho_{[p, q]}\left(A_{s}\right) .
\end{gathered}
$$

Acknowledgements. This paper was supported by the Directorate-General for Scientific Research and Technological Development (DGRSDT).

\section{RefERENCES}

[1] B. Belaïdi, Growth and oscillation theory of $[p, q]$-order analytic solutions of linear differential equations in the unit disc. J. Math. Anal. 3 (2012), 1-11. http://www. ilirias. com/jma/repository/docs/JMA3-1-1. pdf.

[2] B. Belaïdi, Iterated order of meromorphic solutions of homogeneous and non-homogeneous linear differential equations. Romai J. 11 (2015), 33-46. http://rj.romai.ro/arhiva/2015/1/RJv11n1.pdf.

[3] B. Belaïdi, Differential polynomials generated by meromorphic solutions of $[p, q]$-order to complex linear differential equations. Rom. J. Math. Comput. Sci. 5 (2015), 46-62. http://rjm-cs.ro/Belaidi-2015.pdf.

[4] A. Ferraoun and B. Belaïdi, On the $(p, q)$-order of solutions of some complex linear differential equations. Commun. Optim. Theory, 2017 (2017), Article ID 17, 1-23. https://doi.org/10.23952/cot. 2017.17.

[5] G. G. Gundersen, Estimates for the logarithmic derivative of a meromorphic function, plus similar estimates. J. London Math. Soc. (2) 37 (1988), 88-104. https://doi.org/10.1112/jlms/s2-37.121.88.

[6] G. G. Gundersen, Finite order solutions of second order linear differential equations. Trans. Amer. Math. Soc. 305 (1988), 415-429. https://doi.org/10.2307/2001061.

[7] A. A. Goldberg and I. V. Ostrovskii, The distribution of values of meromorphic functions. Irdat Nauk, Moscow, 1970 (in Russian), Transl. Math. Monogr., vol. 236, Amer. Math. Soc. Providence RI, 2008.

[8] K. Hamani and B. Belaïdi, Growth of solutions of complex linear differential equations with entire coefficients of finite iterated order. Acta Univ. Apulensis Math. Inform. 27 (2011), 203-216. http://emis. impa. br/EMIS/ journals/AUA/acta27/Paper21-Acta27-2011.pdf.

[9] W. K. Hayman, Meromorphic functions. Oxford Mathematical Monographs, Clarendon Press, Oxford 1964.

[10] W. K. Hayman, The local growth of power series: a survey of the Wiman-Valiron method. Canad. Math. Bull. 17 (1974), 317-358. https://doi org/10.4153/CMB-1974-064-0.

[11] H. Hu and X. M. Zheng, Growth of solutions to linear differential equations with entire coefficients. Electron. J. Differ. Equations 2012 (2012), 226, 15 pp. https://ejde.math. txstate. edu/Volumes/2012/226/hu.pdf. 
[12] O. P. Juneja, G. P. Kapoor and S. K. Bajpai, On the $(p, q)$-order and lower $(p, q)$-order of an entire function. J. Reine Angew. Math. 282 (1976), 53-67. https://doi.org/10.1515/crl1.1976.282.53.

[13] L. Kinnunen, Linear differential equations with solutions of finite iterated order. Southeast Asian Bull. Math. 22 (1998), 385-405.

[14] I. Laine, Nevanlinna theory and complex differential equations. de Gruyter Studies in Mathematics, 15. Walter de Gruyter \& Co., Berlin, 1993. https://doi.org/10.1515/9783110863147.

[15] L. M. Li and T. B. Cao, Solutions for linear differential equations with meromorphic coefficients of $[p, q]$-order in the plane. Electron. J. Differ. Equations 2012 (2012), 195, 15 pp. https://ejde.math.txstate. edu/Volumes/ 2012/195/li.pdf.

[16] J. Liu, J. Tu and L. Z. Shi, Linear differential equations with entire coefficients of $[p$, $q]$-order in the complex plane. J. Math. Anal. Appl. 372 (2010), 55-67. https://doi.org/10.1016/j.jmaa. 2010.05.014.

[17] M. Saidani and B. Belaïdi, Oscillation of solutions and their arbitrary order derivatives of higher order non-homogeneous LDE. Scientific Publications of the State University of Novi Pazar, Ser. A: Appl. Math. Inform. And Mech. 9 (2017), 103-126. https://scindeks-clanci.ceon.rs/data/pdf/2217-5539/2017/ 2217-55391702103S.pdf.

[18] M. Saidani and B. Belaïdi, On the growth of solutions of homogeneous and non-homogeneous linear differential equations with meromorphic coefficients. Sci. Stud. Res. Ser. Math. Inform. 28 (2018), 131-146. https://pubs. ub. ro/?pg=revues\&rev=s.srsmi\&num=201801\&vol=28\&aid=4817.

[19] M. Saidani and B. Belaïdi, Meromorphic solutions to linear differential equations with entire coefficients of [ $p, q]-$ order. J. Dyn. Syst. Geom. Theor. 16 (2018), 33-53. https://doi.org/10.1080/1726037X. 2017.1413065.

[20] J. Tu and Z. X. Chen, Growth of solutions of complex differential equations with meromorphic coefficients of finite iterated order. Southeast Asian Bull. Math. 33 (2009), 153-164.

[21] J. Tu and T. Long, Oscillation of complex high order linear differential equations with coefficients of finite iterated order. Electron. J. Qual. Theory Differ. Equ. 2009 (2009), 66, 1-13. https://www.math.u-szeged.hu/ejqtde/ p453.pdf.

[22] G. Valiron, Lectures on the General Theory of Integral Functions. translated by E. F. Collingwood, Chelsea, New York, 1949.

[23] H. Y. Xu, J. Tu and Z. X. Xuan, The oscillation on solutions of some classes of linear differential equations with meromorphic coefficients of finite [p,q]-order. Sci. World J. 2013 (2013), Article ID 243873, 8 pages. https: //doi.org/10.1155/2013/243873.

[24] C. C. Yang and H. X. Yi, Uniqueness theory of meromorphic functions. Mathematics and its Applications, 557. Kluwer Academic Publishers Group, Dordrecht, 2003.

[25] M. L. Zhan and X. M. Zheng, Solutions to linear differential equations with some coefficient being lacunary series of $[p, q]$-order in the complex plane. Ann. Differ. Equations 30 (2014), 364-372. 